\title{
Design And Implementation of Gas Turbine Combustion Chamber Control System Based on Wireless Sensor Network
}

\author{
Neda Khan, Rehan Muzammil, M. Salim Beg \\ Department of Electronics Engg. Aligarh Muslim University, Aligarh,India
}

\begin{abstract}
Wireless Sensor Networks (WSNs) are increasingly being used in various applications such as for controlling \& monitoring purposes in industrial processes. Cost effectiveness is the highly prioritized goal of communication protocols \& application design for WSN. This paper discusses the development of a WSN to control \& monitor Gas Turbine (GT) based Combustion Chamber (CC), which includes the design, development \& implementation of a WSN for real time monitoring \& controlling GT based combustion chamber system. The motive behind this work is to totally eliminate the use of control cables. It may be visualized that the sensors are placed around the periphery of the CC of the GT. The regulation of pressure \& temperature is done using a radio module attached to a data acquisition board and the prototype circuits designed. Programming of the sensor nodes and the gateway is done using software. The prototype system developed is based on 2/3 voting logic for tripping the system if the temperature or pressure goes below or beyond the threshold range. This work is an initiative to save control cable costs to make gas-based power plants cost effective since no such approach has been taken regarding WSN application until now.

Keywords: Wireless Sensor Network, Zigbee, Xmesh, Gas Turbine, Combustion Chamber.
\end{abstract}

\section{INTRODUCTION}

WSNs have extremely limited resource like processing speed, storage capacity, communication power and energy supply. It is self healing \& self configuring networks consisting of mobile or static sensor nodes connected wirelessly to form a discretionary topology. WSN consists of spatially distributed autonomous sensors to monitor physical or environmental conditions such as temperature, pressure, sound, vibration, motion or pollutants and to cooperatively pass their data through the network to a main location. Each such sensor network node has typically several parts: a radio transceiver with an internal antenna or connection to an external antenna, a microcontroller, an electronic circuit for interfacing with the sensors power supply and an actuator. Sensor nodes consist of power management module, sensors, microprocessor, memory and radio frequency module [1][10]. The structure of the sensor node is shown in Fig 1.

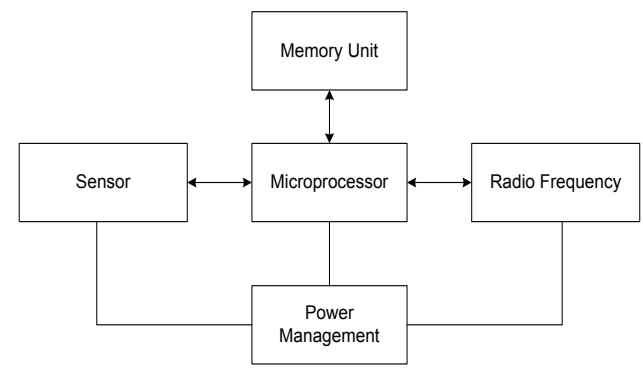

Fig. 1: The structure of Sensor node.

Smart sensor nodes may be attached to varieties of mechanical, thermal, biological, chemical, optical and magnetic sensors to measure properties of the environment. Sensor nodes have limited memory and are typically deployed in difficult-to-access locations. Battery is the main power source in sensor node. Actuators may be incorporated in the sensors depending upon the application and the type of sensors used. WSNs are of 5 different types such as terrestrial WSN, Underground WSN, Underwater WSN, Multimedia WSN and Mobile WSN [1]. Some of the sensors when distributed in the environment comprise WSNs that include cameras as vision sensors, micro-phones as audio sensors. Although the individual sensor's sensing range is limited, WSN can cover a large space by integrating data from many sensors. Wireless sensors can be embedded deeply within machinery, where wired sensors would not be feasible. These sensors could not reach the deeply embedded parts because of being too costly and thus limit the flexibility. It would represent a maintenance problem or would prevent the mobility. For example, Mobile items such as containers can be tagged, as can goods in a factory floor automation system. Smart price tags for foods could communicate with a refrigerator [2][5].

The protocol stack used by the sink, cluster head and sensor nodes, the sensor network protocol stack is much like the traditional protocol stack, with the following layers: application, transport, network, data link, and 
physical. The physical layer is responsible for frequency selection, carrier frequency generation, signal detection, modulation and data encryption. The data link layer is responsible for the multiplexing of data streams, data frame detection, medium access and error control. It ensures reliable point-to-point and point-tomultipoint connections in a communication network. The network layer takes care of routing the data supplied by the transport layer. The network layer design in WSNs must consider the power efficiency, data-centric communication, data aggregation, etc. The transportation layer helps to maintain the data flow and may be important if WSNs are planned to be accessed through the Internet or other external networks. Depending on the sensing tasks, different types of application software can be set up and used on the application layer.

As very little to no work has been done regarding monitoring and control of gas turbine using WSN, hence, motivation of this work is to make the system that cut down the cable costs and save the Indian economy from the loss that is routinely increasing and reflecting it and these two papers [11][12] have used other wireless systems instead of WSN whereas our paper uses WSN to realize the GTCCC along with hundreds of other sensors which may be used in the power plant for various purposes.

This paper is organized as follows: section II illustrates Gas Turbine; section III describes brief details of the WSN used in this work; section IV describes GT Combustion Chamber Control System; section V gives Experimental Setup; section VI gives the real time Results; section VII gives Conclusions.

\section{GAS TURBINE}

Combustion instabilities in industrial gas turbines can produce intolerably large pressure waves and high temperature which lead to fatigue, detachment of components and costly outages and repair. The measurement of dynamic pressure amplitude sand varying temperature ranges within the combustion chamber may be used in condition monitoring and analysis to detect and correct instabilities before they cause serious damage. The control and monitoring process of these power plants deal along with the large setup of control cables. The loss of these control cables due to break down or rodents bite is routinely increasing and reflecting the Indian economy.

A simple gas turbine is comprised of 3 main sections: a compressor, a combustor and a power turbine. The gas turbine operates on the principle of Brayton Cycle, where compressed air is mixed with fuel and burnt under constant pressure conditions. The resulting hot gas is allowed to expand through a turbine to perform work. The various sections of GT combustion chamber has been shown in Fig. 2. There are two possible combustion chamber geometry configurations:

(i) Annular: - A hollow annulus (or ring) wrapped around the circumference of engine. A series of burners are located at equal intervals around the ring.

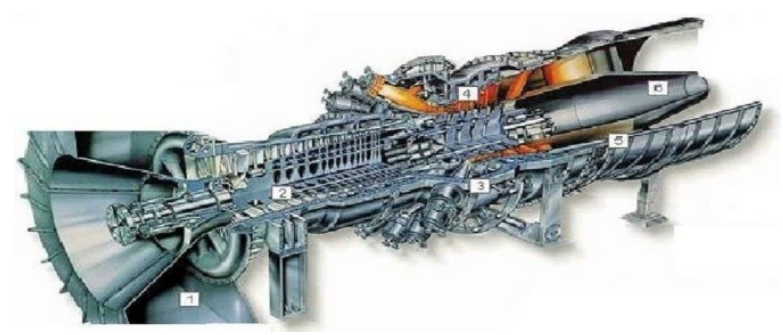

Fig.2: Gas Turbine Combustion Chamber

(ii) Cannular: - It is can-type, or cannular configuration. It is a series of individual, can-shaped combustors placed around the circumference of the engine.

The work reported in this present paper takes into account a cannular type [9].

\section{WIRELESS SENSOR NETWORK}

When all the sensor nodes having the transmission range to be large enough so the data can be directly transmitted to the base station by the sensors, they form a star topology. Multi-hop communication is the most common case for sensor networks. These sensor nodes form some other topology such as cluster tree network and mesh topology. Zigbee based WSN technology of low data rate, low power consumption \& low cost has been used in this present work. Zigbee provides low power connectivity for equipment that needs battery life as long as several months to several years. Zigbee can be implemented in mesh networks larger than is possible with Bluetooth. Zigbee compliant wireless devices are expected to transmit 10-75 meters, depending on the RF environment and the power output consumption required for a given application. It will operate in the unlicensed RF worldwide.

Crossbow developed XMesh for wireless network. XMesh is a full featured multi-hop, adhoc, mesh networking protocol which consists of nodes that wirelessly communicate to each other. They are capable of 
hopping radio messages to a base station where they are passed to a PC or other client. XMesh provides a TrueMesh networking service that is self-organizing \& self-healing. Also XMesh can be configured into various power nodes including HP (High Power), ELP (Extra Low Power) and LP (Low Power). In HP, the node that make up XMesh Network are always on. This is the highest bandwidth mode for transmitting packets to the base station mote. In ELP, ELP achieves the minimum operating power because motes spend almost all of their time in a sleep state. In LP, the nodes in XMesh network which goes into sleep mode when not in use. In this case XMesh has been configured into LP mode [3][8][9].

\section{GT COMBUSTION CHAMBER CONTROL SYSTEM}

The prototype circuit has been designed for GT combustion chamber control system. This setup consists of three computers namely R, S \& T which are individually designed with the same logic and circuits. These computers or systems are

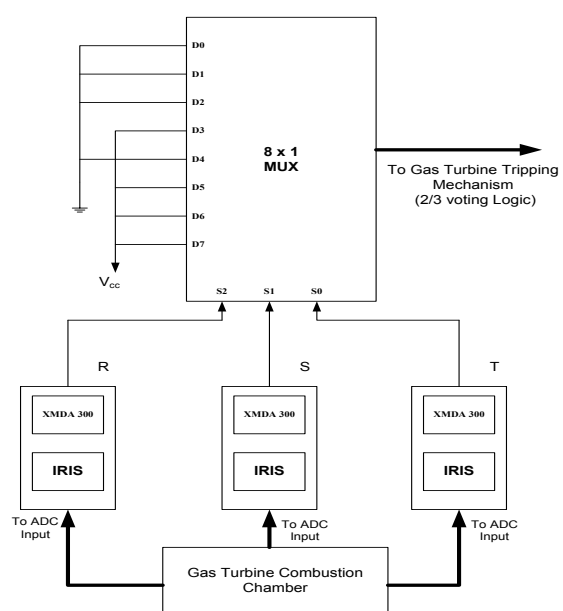

Fig. 3: GT Combustion Chamber Control System

basically depending upon the parameters mainly pressure and temperature where transducers \& thermocouples are used as pressure \& temperature sensors respectively. Considering any of system say R at the individual level, if any of the two sensors either pressure or temperature ones are malfunctioned, the system will get tripped. Likewise $S \& T$ will perform their operation. Following the outputs of these three systems and $2 / 3$ voting logic, the control system will decide to make the system to run or trip. The architecture of Gas Turbine Combustion Chamber control system (GTCCCS) is shown in the Fig. 3 [3][4].

\section{EXPERIMENTAL SETUP}

The idea behind the prototype system is to develop an analogous circuit of GTCCCS circuit which will work at low voltage supply. As our sensor board has an analog line voltage limit of $2.5 \mathrm{~V}$, which motivated us to develop the prototype of GTCCCS. This prototype circuit shows the pressure \& temperature sensing data acquisition circuit of each combustion chamber as shown in Fig. 4. Similar to our real Gas Turbine Combustion Chamber, dc motor here is attached at the output as an analogy of system.

The GT Combustion Chamber Control system includes three parts which are named as prototype circuit, sensor mote and DC motor \& relays.

- Sensor mote consists of XMDA300 sensor board with IRIS module radio.

- Prototype Circuit consists of LEDs in parallel with voltage across variable resistance \& the overloading threshold kept is $2.4 \mathrm{~V}$.

- $\quad$ The DC motor should be turned ON by 5 volt circuit through relay \& 2 relays that work on $5 \mathrm{~V}$ to $30 \mathrm{~V}$.

The prototype circuit further consists of the following two to form a system (say R);

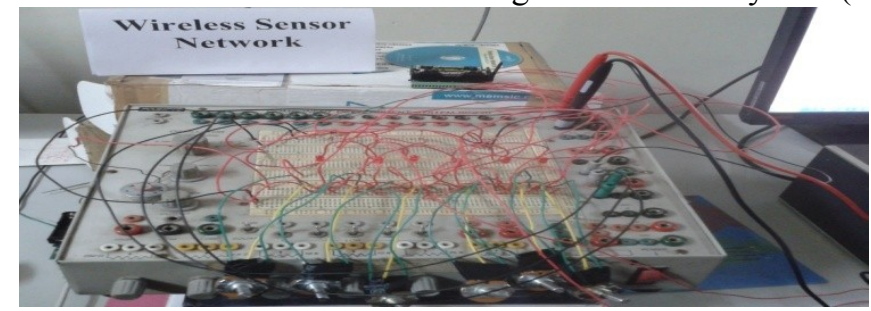

Fig. 4: The Prototype System 


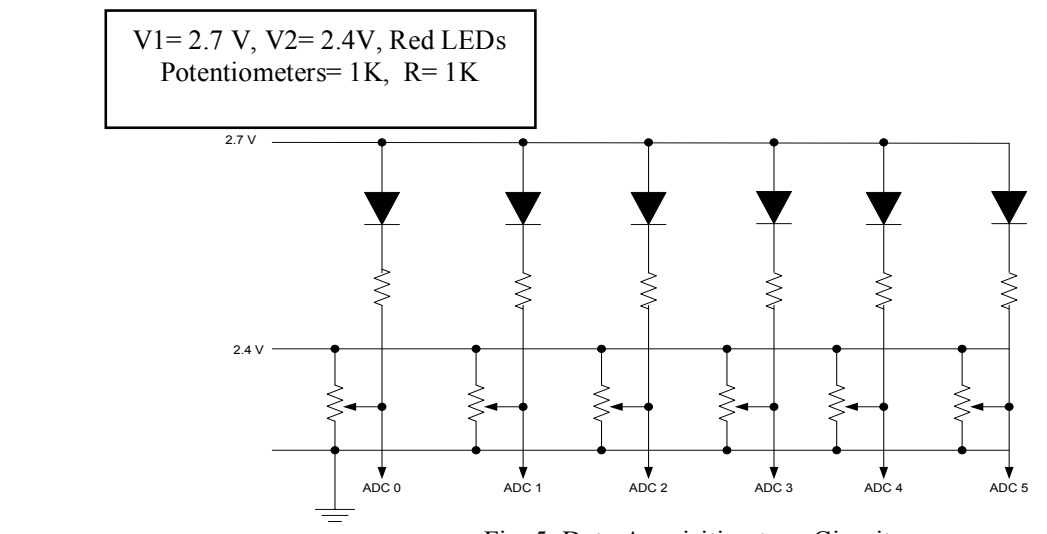

Fig. 5: Data Acquisition type Circuit

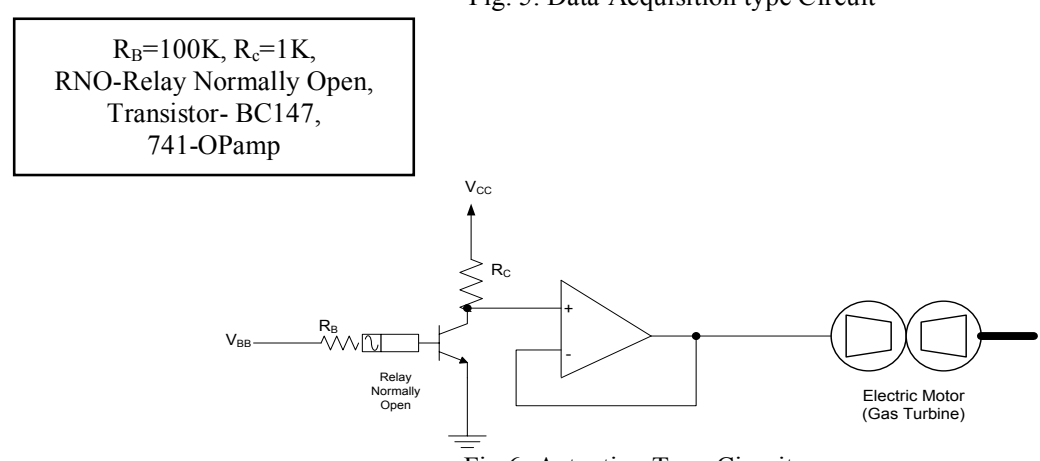

Fig.6: Actuation Type Circuit

- Data Acquisition Type Circuit

- Actuation Type Circuit

Both these circuits are shown in Fig. 5 and Fig. 6 respectively. Likewise the systems S and T are implemented with the same designed prototype circuit.

The Data Acquisition system for GT Combustion Chamber consists of mote which accesses the temperature \& pressure transducer into voltage $\&$ this value of voltage is transmitted to the gateway via the radio for monitoring purpose only. The actuation system does tripping or control of gas turbine system \& it is normally done by relay normally open. There are three potentiometers for temperature sensors $\&$ three for pressure sensors for monitoring the gas turbine. $2 / 3$ voting logic between the three thermocouples $\&$ three pressure transducers are separately practiced.

The prototype system developed will get tripped if any of the ADC lines as shown in Fig. 4 (shown by yellow wires) from " 0 " to "5" is less than $\mathrm{V}_{\text {Th1 }}$ or higher than $\mathrm{V}_{\text {Th2 }}$ (i.e. if the temperature or pressure goes below the minimum or beyond the maximum threshold) and if any of these lines voltage is between $\mathrm{V}_{\mathrm{Th} 1} \& \mathrm{~V}_{\mathrm{Th} 2}$, the system will be back in running mode. The common emitter based circuit with collector \& base resistance and relay normally open as an actuator is used as switch so as to run or trip the system [9]. The truth table is shown in Table I.

$*_{-}$TRIP $={ }^{\prime} 0$ '

Table I: Truth Table of the $8 \times 1$ MUX output

\begin{tabular}{|c|c|c|c|}
\hline \multicolumn{3}{|c|}{ Input } & \multirow{2}{*}{ Output } \\
\hline$s 2$ & $s 1$ & so & \\
\hline 0 & 0 & 0 & $\mathrm{~T}^{*}$ \\
\hline 0 & 0 & 1 & $\mathrm{~T}$ \\
\hline 0 & 1 & 1 & $\mathrm{R}^{* *}$ \\
\hline 0 & 1 & 0 & $\mathrm{~T}$ \\
\hline 1 & 1 & 0 & $\mathrm{R}$ \\
\hline 1 & 1 & 1 & $\mathrm{R}$ \\
\hline 1 & 0 & 1 & $\mathrm{R}$ \\
\hline 1 & 0 & 0 & $\mathrm{~T}$ \\
\hline
\end{tabular}

** RUN $=$ ' 1 ' 


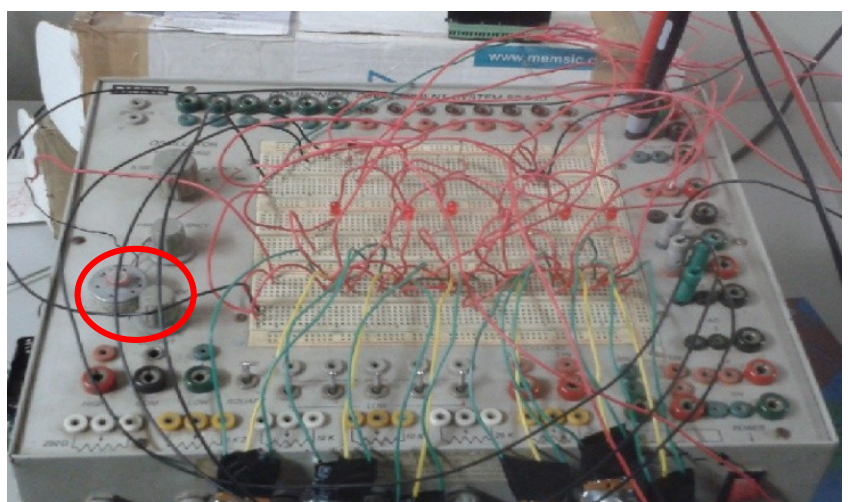

Fig.7: Setup showing the Running mode during voltage between $V_{T h 1} \& V_{T h 2}$

\section{RESULTS}

The two modes of this system are running and tripping

- Running Mode: When the voltage at the ADCs is between $\mathrm{V}_{\mathrm{Th} 1}$ \& $\mathrm{V}_{\mathrm{Th} 2}$, this situation is shown in circuit diagram in Fig. 7.

- Tripping Mode for R, S and T:According to the conditions applied, mote no. 20, 21 and 22 namely system $\mathbf{R}, \mathbf{S}$ and $\mathbf{T}$ respectively are tripped. The tripping mode for $\mathrm{R} \& \mathrm{~S}, \mathrm{R} \& \mathrm{~T}$ and $\mathrm{S}$ and $\mathrm{T}$ are shown in Fig. 8, Fig. 9 and Fig. 10 respectively.
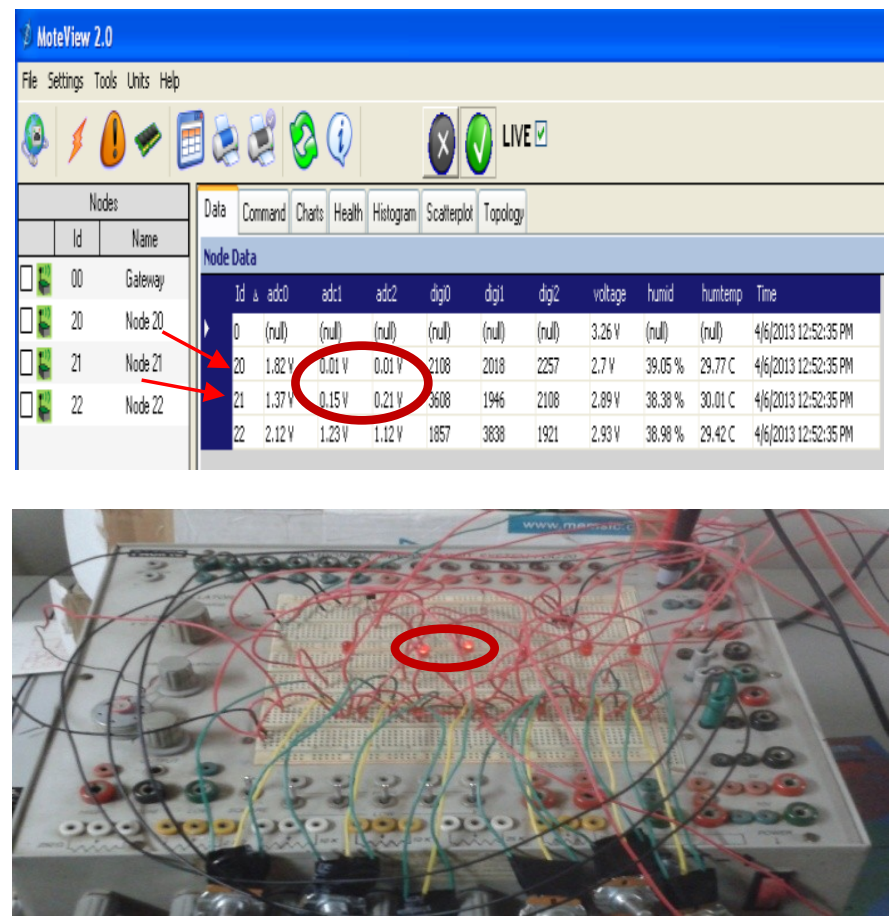

Fig.8. Tripping of System R \& S shown in the MoteView and over the setup 

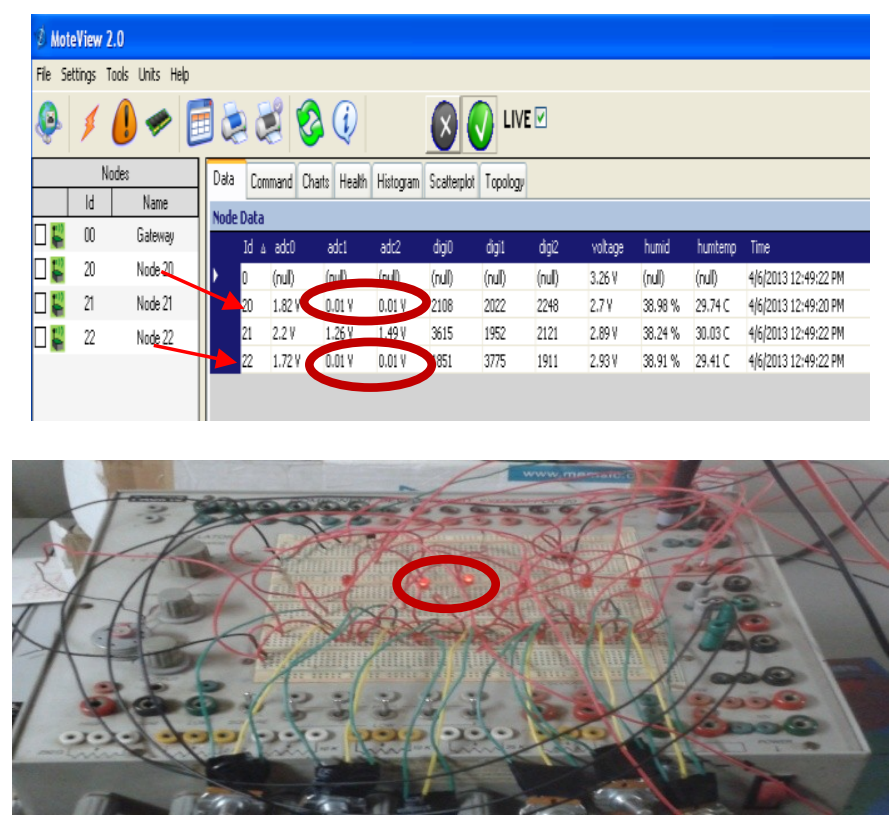

Fig. 9:Tripping of System R \& T shown in the MoteView and over the setup
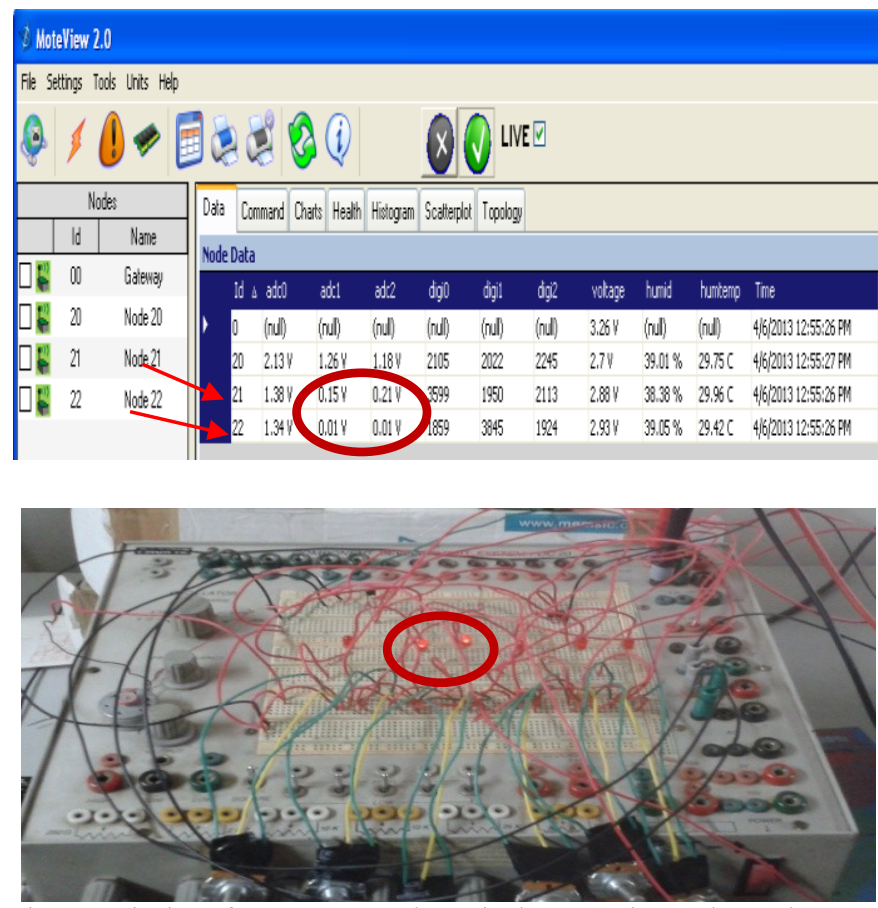

Fig. 10: Tripping of System S \& T shown in the MoteView and over the setup

The system application changes its reading around every second. The ADCs are used as data acquisition system which accepts these voltages from the sensors. This voltage coming from the potentiometer is assumed to be coming from the pressure \& temperature sensors. Because of the MoteView limitations, only three of the ADCs lines are shown in the MoteView. Thus, if the system is in tripped condition, the effect of the malfunctioned pressure sensors are not able to be displayed over the MoteView although its parameters are mapped to the voltage of potentiometers.

\section{Conclusions}

The complete system has been designed \& implemented including R, S \& T. Following the system $2 / 3$ voting logic among these individual systems, the system comes in condition of running or tripping. Individually also the systems R, S \& T depend upon the $2 / 3$ voting logic among the thermocouples $\&$ transducers. The temperature $\&$ pressure sensors are formed by using the potentiometer \& power supply which is assumed to be 
the sensors; since there are no pressure or temperature sensors on the XMDA300C which can be used for this purpose. The system has been actually tested and not simply designed.

\section{References}

[1] V. Madan andS.R.N. Reddy, "Review of Wireless Sensor Mote Platforms", VSRD-IJEECE, Vol.2(2), 2012, pp. 51-55.

[2] G. Zhao and C.A. Davis, "Wireless Sensor Networks for Industrial Process Monitoring and Control: A Survey", ISSN 1943-3581, Vol.3, No.1, 2011, pp. 46-63

[3] F. Huang and T. Sun, "Design \& Implementation of Radiation Dose Monitoring System Based on Wireless Sensor Network", International Conference on Future Information Technology IPCSIT, 2011, pp. 430-434

[4] K. Sha, W. Shi and O.Watkins, "Using Wireless Sensor Networks For Fire Rescue Applications: Requirements \& Challenges", IEEEInternational Conference on Electro/Information Technology, May 2006, pp.239-244Y.

[5] J.A. Gutierrez, B.D. Durocher and B. Lu, “Applying Wireless Sensor Networks in Industrial Plant Energy Evaluation \& Planning Systems", IEEE International Conference on Pulp and Paper Industry Technical Conference, 2006, pp. 1-7.

[6] A. Nanda, B. Dinda, R. Rath and S. Pradhan, "Wireless Sensor Network for Super Cyclone prediction using Artificial Neural Network “, Asian Journal of Computer Science and Information Technology 1:2, 2011, pp. 30-33.

[7] S. Taruna,K. Jain and G.N. Purohit, "Application Domain of WSN:- A Paradigm in Developed and Developing Countries", IJCSI International Journal of Computer Science Issues, Vol.8, Issue 4, No.2, July 2011, pp. 611-617.

[8] S. Stankovic, "Medical Applications Based on Wireless Sensor Networks", unpublished.

[9] Neda Khan, "Use of Wireless Sensor Network for Fault Tolerant Control of Gas Turbine", M.Tech. (Electronics Engg.) Dissertation, AMU, 2013.

[10] R.Shamim, M.Salim Beg, R.Muzammil, "Implementation of a Wireless Sensor Network for Real Time Temperature Monitoring and Regulation", Proceedings of National Conference on Advances in Wireless Cellular Telecom, New Delhi, April 2011, pp. 112-118.

[11] A.D.DeHennis and K.D. Wise, "A Wireless Microsystem for the Remote Sensing of Pressure, Temperature and Relative Humidity", Journal of Microelectromechanical system, Vol. 14, No. 1, Feb 2005, pp. 12-22.

[12] M.A. Fonseca, J.M. English, M. Von Arx and M.G. Allen, "Wireless Micromachined Ceramic Pressure sensor fr High Temperature Applications”, Journal of Microelectromechanical systems, Vol. 11, No. 4, Aug 2002, pp. 337-343. 